\title{
Star complexes and stellar populations in NGC 6822
}

\section{Comparison with the Magellanic Clouds}

\author{
A. Karampelas ${ }^{1}$, A. Dapergolas ${ }^{2}$, E. Kontizas ${ }^{2}$, E. Livanou ${ }^{1}$, M. Kontizas ${ }^{1}$, I. Bellas-Velidis ${ }^{2}$, and J. M. Vílchez ${ }^{3}$ \\ 1 Department of Astrophysics, Astronomy \& Mechanics, Faculty of Physics, University of Athens, 15783 Athens, Greece \\ e-mail: ankaramp@phys.uoa.gr \\ 2 Institute for Astronomy and Astrophysics, National Observatory of Athens, PO Box 20048, 11810 Athens, Greece \\ 3 Instituto de Astrofísica de Andalucía (CSIC), Apartado 3004, 18080 Granada, Spain
}

Received 3 October 2008 / Accepted 27 January 2009

\section{ABSTRACT}

\begin{abstract}
Aims. The star complexes (large scale star forming regions) of NGC 6822 were traced and mapped and their size distribution was compared with the size distribution of star complexes in the Magellanic Clouds (MCs). The spatial distributions of different age stellar populations were compared with each other.

Methods. The star complexes of NGC 6822 were determined by using the isopleths, based on star counts, of the young stars of the galaxy, using a statistical cutoff limit in density. In order to map them and determine their geometric properties, an ellipse was fitted to each distinct region satisfying this minimum limit. The Kolmogorov-Smirnov statistical test was used to study possible patterns in their size distribution. Isopleths were also used to study the stellar populations of NGC 6822.

Results. The star complexes of NGC 6822 were detected and a list of their positions and sizes was produced. Indications of hierarchical star formation, in terms of spatial distribution, time evolution and preferable sizes were found in NGC 6822 and the MCs. The spatial distribution of the various age stellar populations has indicated traces of an interaction in NGC 6822, dated before $350 \pm 50$ Myr.
\end{abstract}

Key words. galaxies: stellar content - galaxies: structure - galaxies: individual: star complexes

\section{Introduction}

Stars are considered to be born inside stellar groupings, the lowest in size being $\mathrm{OB}$ associations. Bigger groupings, containing star clusters, OB associations, HII regions and individual bright stars, are star complexes, with an age of up to $\sim 100$ Myr (Efremov 1978, 1979). Star complexes seem to be part of a continuous star formation hierarchy, in which stars form in hierarchically clustered systems from sub-parcec to kiloparcec scales, following the hierarchical distribution of the gas (Elmegreen \& Efremov 1996). The study of the positions and ages of Cepheid variables in the Large Magellanic Cloud (Elmegreen \& Efremov 1996) and star clusters of the same galaxy (Efremov \& Elmegreen 1998) results in the finding that star formation is not only hierarchical in space, but in time as well. Larger star forming regions have higher average ages than smaller regions, forming stars over a longer period. The above suggestions (hereafter the Efremov \& Elmegreen model) are confirmed in subsequent studies of the Large Magellanic Cloud (Maragoudaki et al. 1998; Livanou et al. 2006) and the Small Magellanic Cloud (Maragoudaki et al. 2001; Livanou et al. 2007). According to their sizes, star complexes are empirically divided (Efremov 1987; Maragoudaki et al. 1998; Livanou et al. 2007) into stellar aggregates, stellar complexes and stellar supercomplexes, with sizes from $150 \mathrm{pc}$ to $300 \mathrm{pc}, 300 \mathrm{pc}$ to $1 \mathrm{kpc}$ and greater than $1 \mathrm{kpc}$, respectively. Their properties seem to be universal, revealing their importance in studies of star formation in galaxies and in galaxy evolution.

NGC 6822 is a Local Group dwarf irregular galaxy, discovered by Barnard in 1884. It is located in a relatively isolated position of the Local Group of Galaxies, being a member of the so-called "Local Cloud" of dwarf Irregulars (Mateo 1998), at a distance of $500 \mathrm{kpc}$. The general properties of NGC 6822 are given in Table 1.

The stellar content of NGC 6822 forms a stellar halo extended more than one degree, with the young population in a bar-like structure (PA $\sim 10^{\circ}$, Hodge 1977) and the old population elliptically distributed (Demers et al. 2006). The star formation rate (SFR) of NGC 6822 is supposed to have been relatively low and constant during the past, but increased recently, especially in the bar. Gallart et al. (1996b) argue for an increasing SFR during the last $400 \mathrm{Myr}$ in comparison to the past, which is more obvious for the last 100-200 Myr, mainly in the bar, while Wyder (2001) detects the beginning of this increase back to 600 Myr. Furthermore, Hodge (1980) has discovered an increased star cluster formation rate during the last 75-100 Myr and Skillman et al. (1989) traced a low value of the N/O ratio, which is characteristic of low metallicity, active star forming dwarf galaxies.

Almost vertical to the elliptical structure of the old stellar population lies the highly disturbed elongated disk of atomic hydrogen (PA $\sim 130^{\circ}$, Weldrake et al. 2003). The most striking features of the gas disk are the $1 \mathrm{kpc}$-size giant hole (Hodge et al. 1991; de Blok \& Walter 2000), the SE tidal arm and the NW cloud (de Blok \& Walter 2000). An upper limit of $130 \mathrm{Myr}$ is set for the kinematical age of the giant hole, while the same quantity is estimated for the tidal arm to be around $140 \mathrm{Myr}$ (de Blok \& Walter 2003, 2006).

The NW cloud is considered by de Blok \& Walter (2000, 2006) to be a companion galaxy of NGC 6822. This 

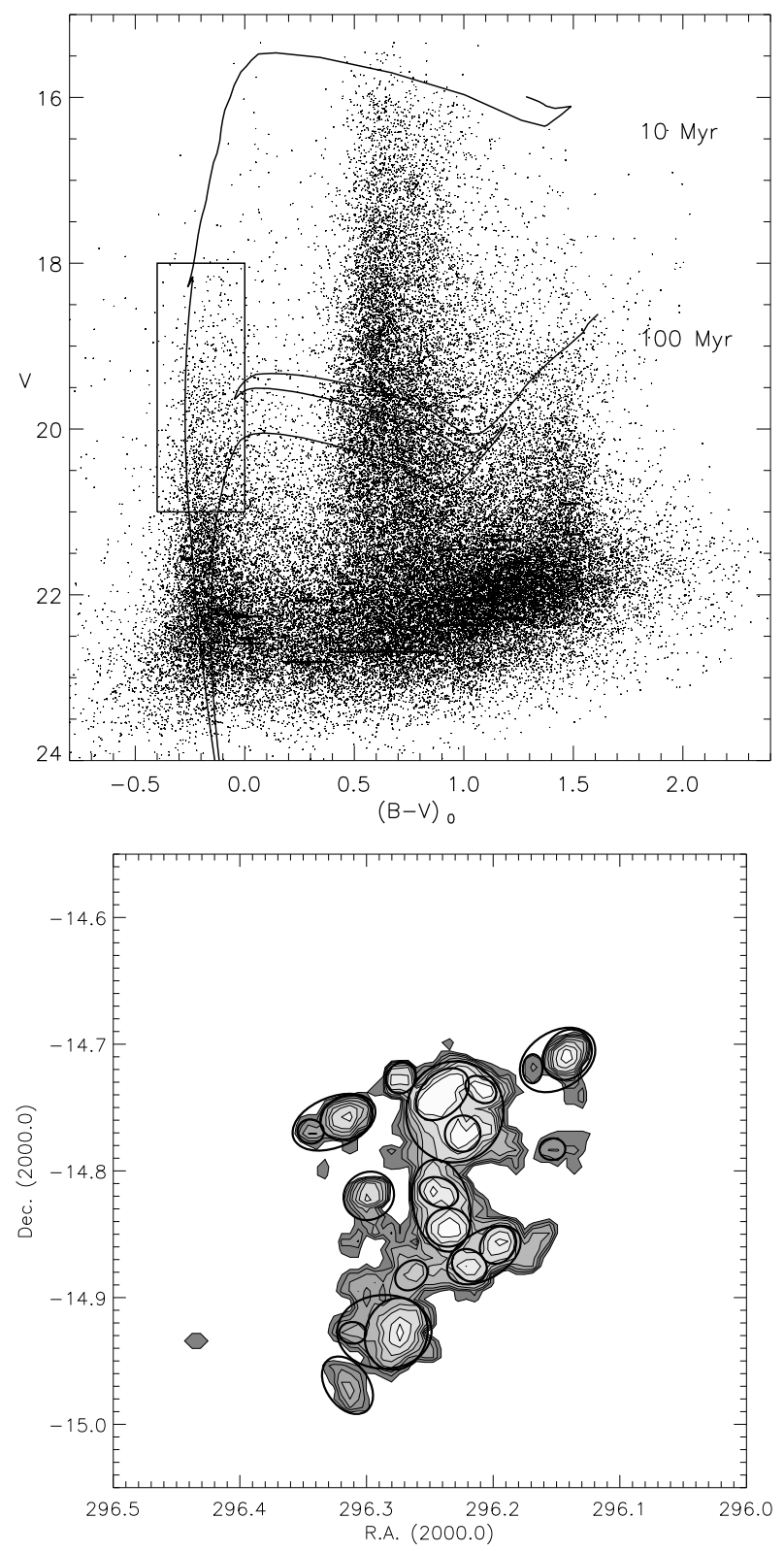

Fig. 2. Upper: CMD diagram of NGC 6822 and photometric uncertainties in colour and magnitude of 30 stars randomly selected from the stellar catalogue. The rectangle includes the young stellar population that was used for the detection of star complexes, and its characteristic theoretical isochrones. Lower: detected star complexes of NGC 6822 (ellipses) plotted over the isopleths of the surface stellar density map of the young stars.

of the stars according to their relative location in the camera (Fig. 1) provides an estimation of the completeness of the data. The stellar catalogue is quite complete until $V=22$.

\section{Determination of star complexes}

The colour-magnitude diagram (CMD) of NGC 6822 was constructed using the "Local Group Survey" stellar catalogue and adopting the values of 23.49 mag for the distance modulus (Gallart et al. 1996a) and $0.25 \mathrm{mag}$ for the reddening (Massey et al. 2007). In order to select young main sequence stars with an age of no more than about 100 Myr to study star complexes, there is a need for colour and magnitude cutoff limits to define a proper selection slice of the CMD. After properly fitting theoretical isochrones (Girardi et al. 2002) of $Z=0.004$ to the main sequence, we adopted the ranges $18 \leq V \leq 21$ and $-0.4 \leq(B-V)_{0} \leq 0$ for the magnitude and the colour, respectively. The CMD diagram of NGC 6822, with the selected slice and its characteristic theoretical isochrones are shown in Fig. 2. In the same figure the photometric uncertainties are illustrated by the colour and magnitude error bars of 30 stars randomly selected from the stellar catalogue (see Sect. 2).

In order to properly use our data to identify the star complexes, we produced a stellar density map of the young stars of NGC 6822, which was constructed by counting stars in bins of $23.5^{\prime \prime} \times 23.5^{\prime \prime}\left(57 \times 57 \mathrm{pc}^{2}\right)$ and by applying a $3 \times 3$ $\left(70.5^{\prime \prime} \times 70.5^{\prime \prime}\right)$ mean-smoothing. The binning mentioned above was chosen after several tests as the best compromise between spatial resolution and statistical significance.

To detect the star complexes of NGC 6822 we computed a density background. By using the pixels of the density map with a value greater than zero we localize the background calculation so as to be characteristic of the star formation and to avoid areas outside the galaxy which artificially lower the background value. The calculation of the background was carried out by using the $\sigma$-clipping method with respect to the median value. The median and standard deviation values are initially computed for all the (non zero) pixels. Then, pixels with values higher than median $+3 \sigma$ are excluded and median and $\sigma$ are recalculated. This procedure continues until convergence, usually after very few iterations. The final median $+3 \sigma$ value is considered to be the minimum density cutoff limit.

In order to map the star complexes of NGC 6822, we fitted ellipses to the external isopleth of each distinct structure or substructure above the minimum density cutoff limit. The isopleths were drawn from this limit to the maximum value of the density map with a step of one $\sigma$ and a density range of more than two $\sigma$, to avoid the selection of random or controversial concentrations. In some cases optimization led us to the compromise of taking into account two subsequent isopleths to fit an ellipse. In Fig. 2 the ellipses that represent the star complexes of each galaxy are overplotted on the isopleths. The center coordinates and major axis length of the ellipses are considered to be the position and size of the star complexes they outline, respectively. Table 2 lists the center coordinates $\left(\alpha_{2000}, \delta_{2000}\right)$ and the size of each one of the detected star complexes of NGC 6822, sorted in descending order of the right ascension. They are conventionally named NGC6822-SC-i, where SC stands for Star Complex and $i$ ranges from 1 to 24 .

Figure 3 shows the spatial distribution of young main sequence stars of different maximum age, ranging from $40 \mathrm{Myr}$ to $100 \mathrm{Myr}$ in time bins of $15 \mathrm{Myr}$. The last plot shows the ellipses outlining the detected star complexes of NGC 6822. Structures younger than $\sim 55 \mathrm{Myr}$ are clearly less extended than structures containing stars with ages up to $\sim 100 \mathrm{Myr}$.

\section{Size distribution of star complexes - comparison with the Magellanic Clouds}

Since the star complexes have been detected, what is examined next is the distribution of their size (as it was defined in Sect. 3). In Fig. 4 the histogram of the size distribution of detected star complexes of NGC 6822 is shown, with the same plots for the Large Magellanic Cloud (Maragoudaki et al. 1998; Livanou et al. 2006), based on optical observations, and the Small Magellanic Cloud (Livanou et al. 2007), based on infrared observations, with a common bin size of $100 \mathrm{pc}$. A few regions 
Table 2. List of NGC 6822 detected star complexes, in descending order of right ascension.

\begin{tabular}{lccc}
\hline \hline Name & $\begin{array}{c}\alpha_{2000}{ }^{a} \\
(\mathrm{~h} \mathrm{~m} \mathrm{~s})\end{array}$ & $\begin{array}{c}\delta_{2000}{ }^{a} \\
\left({ }^{\prime}{ }^{\prime \prime}\right)\end{array}$ & $\begin{array}{c}\text { Size }^{a} \\
(\mathrm{pc})\end{array}$ \\
\hline NGC 6822-SC-1 & 194522.58 & -144608.15 & 182 \\
NGC 6822-SC-2 & 194518.16 & -144541.62 & 600 \\
NGC 6822-SC-3 & 194515.80 & -144528.33 & 372 \\
NGC 6822-SC-4 & 194515.60 & -145809.05 & 440 \\
NGC 6822-SC-5 & 194514.62 & -145540.15 & 186 \\
NGC 6822-SC-6 & 194511.57 & -144910.96 & 356 \\
NGC 6822-SC-7 & 194508.62 & -145537.20 & 650 \\
NGC 6822-SC-8 & 194506.36 & -145543.11 & 504 \\
NGC 6822-SC-9 & 194505.48 & -144336.30 & 220 \\
NGC 6822-SC-10 & 194503.52 & -145256.50 & 240 \\
NGC 6822-SC-11 & 194458.30 & -144900.62 & 276 \\
NGC 6822-SC-12 & 194457.81 & -144938.96 & 643 \\
NGC 6822-SC-13 & 194457.62 & -144421.98 & 425 \\
NGC 6822-SC-14 & 194456.54 & -145040.89 & 302 \\
NGC 6822-SC-15 & 194455.26 & -144512.13 & 699 \\
NGC 6822-SC-16 & 194453.78 & -144614.05 & 251 \\
NGC 6822-SC-17 & 194452.51 & -145228.49 & 256 \\
NGC 6822-SC-18 & 194450.34 & -144408.74 & 232 \\
NGC 6822-SC-19 & 194449.75 & -145159.00 & 561 \\
NGC 6822-SC-20 & 194447.10 & -145131.00 & 275 \\
NGC 6822-SC-21 & 194440.52 & -144309.73 & 200 \\
NGC 6822-SC-22 & 194436.78 & -144658.26 & 179 \\
NGC 6822-SC-23 & 194435.79 & -144244.68 & 561 \\
NGC 6822-SC-24 & 194433.93 & -144229.95 & 371 \\
\hline
\end{tabular}

${ }^{a}$ Positions and sizes come from the center coordinates and major axis length of the ellipses fitted on the star complexes, respectively.

of the MCs with size $>900 \mathrm{pc}$ have been excluded for reasons of homogeneity and statistical significance. The MCs were chosen for comparison for two reasons: (a) they are our nearest neighbors in the Local Group and their star complexes are well investigated, and (b) these complexes were detected, in principal, with the same method we use in this paper. In Fig. 5 the sizes of the detected star complexes of NGC 6822 and the MCs are presented, sorted in ascending order. The error for each size value was assumed to be $10 \%$ of this value for NGC 6822 (this work) and the Magellanic Clouds (Livanou et al. 2006, 2007). These plots aim to contribute in a supplementary way to the histograms described above, since they could better reveal the limits, if any, between different size groups. When carefully observing these diagrams, two main size groupings can be distinguished for the three galaxies. The first group ranges from $\sim 150 \mathrm{pc}$ to $300-400 \mathrm{pc}$ and the second up to $\sim 800 \mathrm{pc}$. We refer to them as Group I and Group II. In order to investigate whether these size groupings are indicative of preferable size ranges of star formation in these galaxies, we compared them statistically.

If an apparently distinct group is indeed distinct, it is assumed that the size values it contains should be distributed in a uniform random way. Similarly, if we combine two true distinct groups, which are self-characterized by a uniform random distribution $\left(U_{R}\right)$, their combination should not be characterized by this property any longer. If it could maintain this property, then these two groupings would actually be one, in contradiction with the hypothesis that they are two different groups. So, we compared the two main apparent groupings and their combination with an equal number of uniformly random size values, limited to the same range, for NGC 6822 and the Magellanic Clouds. The comparison was repeated a thousand times to limit the impact of some possible extreme cases of random distributions.
Having to compare two equally unknown data sets of continuous data as a function of a single variable (the size), the twotailed Kolmogorov-Smirnov (K-S) test is the most appropriate. The Null Hypothesis is that the two data sets are drawn from the same distribution. We performed these tests and compared the mean value of the significance level for each pair. Low values of this probability indicate that the two data sets are significantly different, while high values indicate that they are probably consistent with a single distribution. The results for NGC 6822 and the Magellanic Clouds are listed in Table 3.

As an extra control and verification of the procedure described above we did the following: (a) we compared two uniform random distributions a thousand times to check the mean value of the significance level, which was $52.7 \%$. This can be considered as a satisfactory value for two data sets, one being random, of being drawn from the same distribution; (b) we cut the first grouping of the LMC in half and found no consistency between these sub groupings $(0 \%$ significance level). This is a sufficient indicator of restriction to the apparent size groupings.

The derived values of significance levels show that the apparent size groupings of the detected star forming regions could be real distinct groupings: when we compare the size values of Group I with an equal number of uniformly random size values, limited to the same range, they seem to be drawn from the same distribution. Similarly, when we compare the size values of Group II with the corresponding uniform random size values, again the null hypothesis seems to be correct. This is not the case when we consider Group I and Group II as one group and compare them with the corresponding uniform random distribution. Note that Group I is considered to range up to $\sim 300-350 \mathrm{pc}$ for NGC 6822 and up to $\sim 350-400$ pc for the MCs. Trials of the Kolmogorov-Smirnov test close to these limits do not change the results significantly. This difference in the upper limit of the first Group between NGC 6822 and the MCs could be due to selection effects (optical or infrared data) and size determination uncertainties.

\section{Stellar populations}

As described in Sect. 3, a proper selection of the young (age $\leq 100 \mathrm{Myr}$ ) MS stars from the CMD allowed us to produce the spatial distribution of the star complexes and determine them to further investigate some of their geometrical properties. Proceeding the same way, we selected stellar populations of various ages to compare their spatial distribution. The final selection was the result of several tests aiming to trace differences in the spatial distribution of stellar populations, and to cover the most important features of the Colour-Magnitude Diagram, like the Main Sequence and the Red Giant Branch. Figure 6 shows the selected regions from the CMD outlined on it. The overplotted 300, 350 and $400 \mathrm{Myr}$ theoretical isochrones are used to illustrate the separation between the young and the older populations of NGC 6822 (see also Fig. 7). Table 4 lists the colour, magnitude and age limits and the dominant stellar component of the selected CMD slices.

Following the steps described in Sect. 3, density maps were constructed by counting stars in bins of $23.5^{\prime \prime} \times 23.5^{\prime \prime}$ and by applying a $3 \times 3$ mean-smoothing. Isopleths were drawn for densities higher than the background level, computed using the $\sigma$-clipping method relative to the median value. Figure 7 quotes the spatial distribution of the different stellar populations.

The slices I, III and V, containing young stars with an age of less than roughly $400 \mathrm{Myr}$, present the characteristic N-S barlike structure of the young stellar content of NGC 6822. On the 

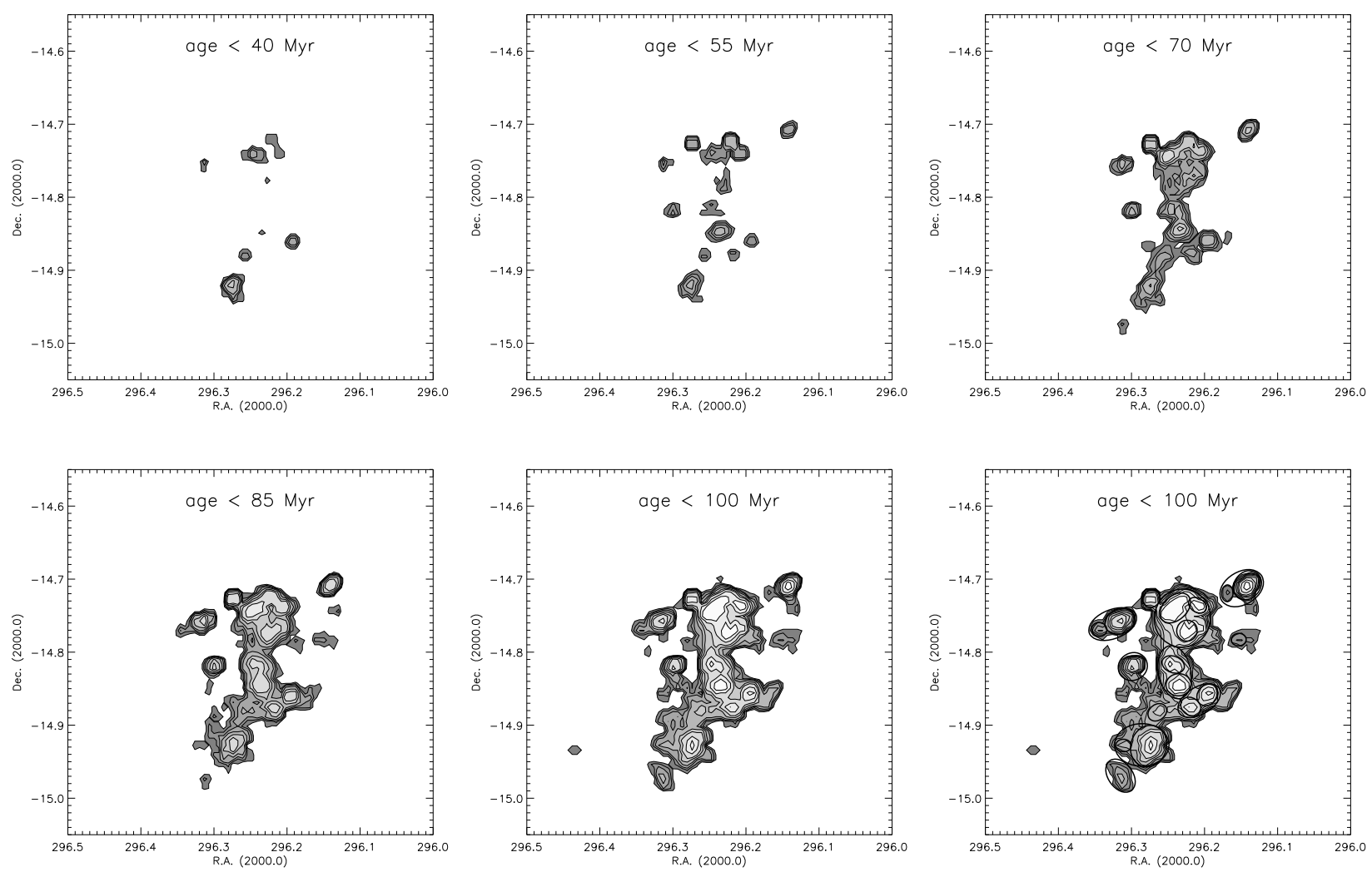

Fig. 3. Spatial distribution of young Main Sequence stars of different maximum age. The last plot shows the ellipses outlining the detected star complexes of NGC 6822 (Table 2).
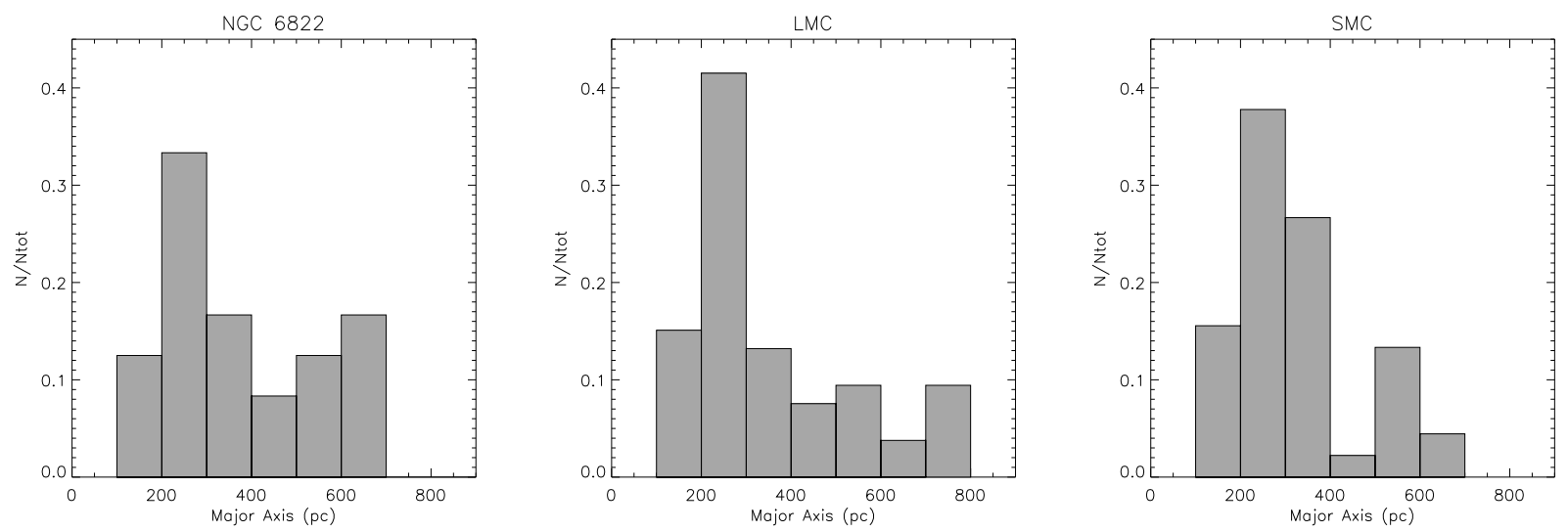

Fig. 4. Histograms of the size distribution of detected star forming regions of NGC 6822 and the Magellanic Clouds, with a bin size of 100 pc.

other hand, the slices II, VI and IX, containing older stars with an age of more than roughly $300 \mathrm{Myr}$, present the characteristic NESW ellipsoidal spatial distribution of the old stellar content of NGC 6822. This leads to a subdivision of the stellar populations of this galaxy into two different stellar components, according to their spatial distribution. The first one contains stars younger than $350 \pm 50 \mathrm{Myr}$, while the second one contains stars older than this age. In Fig. 2, the theoretical isochrones of 300, 350 and $400 \mathrm{Myr}$ illustrate this subdivision. Finally, slices IV, VII and VIII are dominated by background stars of our Galaxy and they do not have an adequate number of NGC 6822 stars to produce any particular structure.

After observing the elliptical spatial distributions of slices II, VI and IX, it is clear that there are two distinct components in the $\mathrm{NE}$ and SW regions of the camera, even though old populations are supposed to be dominated by the long-lasting effects of stellar mixing, resulting in smooth features. This is due to the fact that red and faint stars are expected to appear mainly in the NE and SW regions of the camera due to variations of the PSF and the color responses, affecting the spatial distribution, as it was mentioned in Sect. 2. Nevertheless, the general direction of the old stellar ellipse is probably unaffected, being in agreement with the Red Giants' distribution found by Demers et al. (2006).

\section{Discussion}

\subsection{Hierarchical star formation}

The spatial distribution of the star complexes of NGC 6822 , which have been detected as described above, seem to follow 

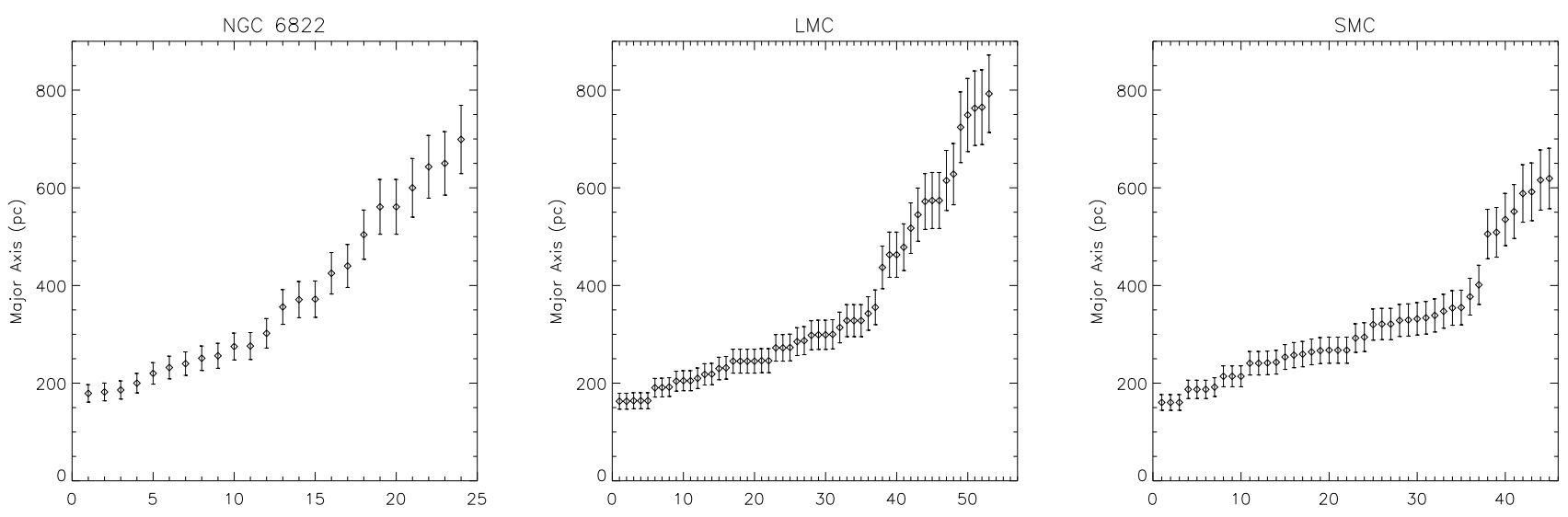

Fig. 5. Sizes of the detected star forming regions of NGC 6822 and the Magellanic Clouds, sorted in ascending order.

Table 3. Significance levels (\%) of the Kolmogorov-Smirnov test for NGC 6822 and the Magellanic Clouds.

\begin{tabular}{lccc}
\hline \hline & Group I & Group II & Group I+II \\
& $U_{R}$ & $U_{R}$ & $U_{R}$ \\
\hline NGC 6822 & 72.7 & 72.9 & 26.2 \\
LMC & 49.7 & 64.3 & 0.2 \\
SMC & 48.5 & 72.5 & 2.1 \\
\hline
\end{tabular}

Column 1: Galaxy. Columns 2-4: Significance levels for the different pairs of size ranges.

the hierarchical star formation scenario, in terms of the distribution of star forming regions in space and their evolution in time. Star complexes are mainly found inside larger star complexes (Fig. 2). Additionally, structures younger than 55 Myr are clearly less extended than structures containing stars with age up to $\sim 100 \mathrm{Myr}$ (Fig. 3). These findings are in agreement with the Efremov \& Elmegreen model, where star complexes are part of a continuous star formation hierarchy, following the hierarchical distribution of the gas (Elmegreen \& Efremov 1996), and star formation proceeds slower in larger star forming regions than in smaller ones (Elmegreen \& Efremov 1996; Efremov \& Elmegreen 1998). Similar conclusions were drawn from previous studies of the LMC (Maragoudaki et al. 1998; Livanou et al. 2006) and the SMC (Maragoudaki et al. 2001; Livanou et al. 2007). However, it is not clear from this study whether the first group of detected star complexes, extended from $\sim 150 \mathrm{pc}$ to $\sim 300-400 \mathrm{pc}$, is characterized by lower average ages than the second one, extended from $\sim 400$ pc to $\sim 800$ pc. Nevertheless, it is clear that younger star forming regions are found to be more concentrated than older ones.

Moreover, the presence of distinct groupings favors the existence of hierarchical star formation, in terms of preferable sizes, in NGC 6822 and the Magellanic Clouds. There is probably consistency with the empirically extracted hierarchy of stellar aggregates, stellar complexes and stellar supercomplexes, within a small range in the size limits between the first two groupings. From the lower scale groupings to the higher, size range increases while the number of star complexes decreases. This conclusion refers to young stellar structures and it remains to be tested also for the smallest ones (OB associations). These structures were not found due to resolution restriction of the observations. If the above scheme is correct and OB associations consist of another distinct group, it could be assumed that they would span a narrower size range and they would contain many more

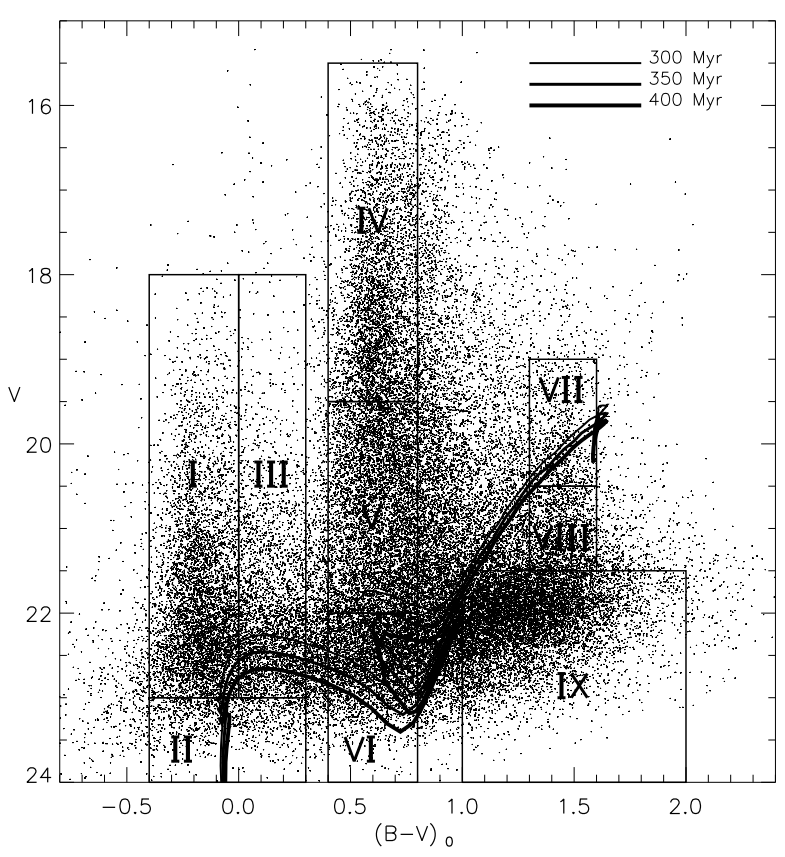

Fig. 6. The regions of the colour-magnitude diagram of NGC 6822 selected to study the respective stellar populations, and the 300,350 and $400 \mathrm{Myr}$ theoretical isochrones that roughly separate the young and the older populations of NGC 6822 .

members. Both have been confirmed observationally in the MCs (Livanou 2007, Ph.D. Thesis).

Assuming that there are preferable size groupings, it is quite clear that they cannot be detected easily from the histograms of the size distribution of star complexes, especially in the case of the LMC. This is not surprising, since there is usually a kind of arbitrariness in selecting the histogram's bins and binning is itself a loss of information. What they can offer most is to indicate the peak values of the size distributions. On the other hand, diagrams with sizes sorted in ascending order together with the Kolmogorov-Smirnov statistical test, seem capable of visualizing groupings, stochastically study their self-consistency and reveal their limits, providing both a qualitative and quantitative approach. Having carried out this procedure, histograms of the size distribution of detected star complexes could be re-examined. 

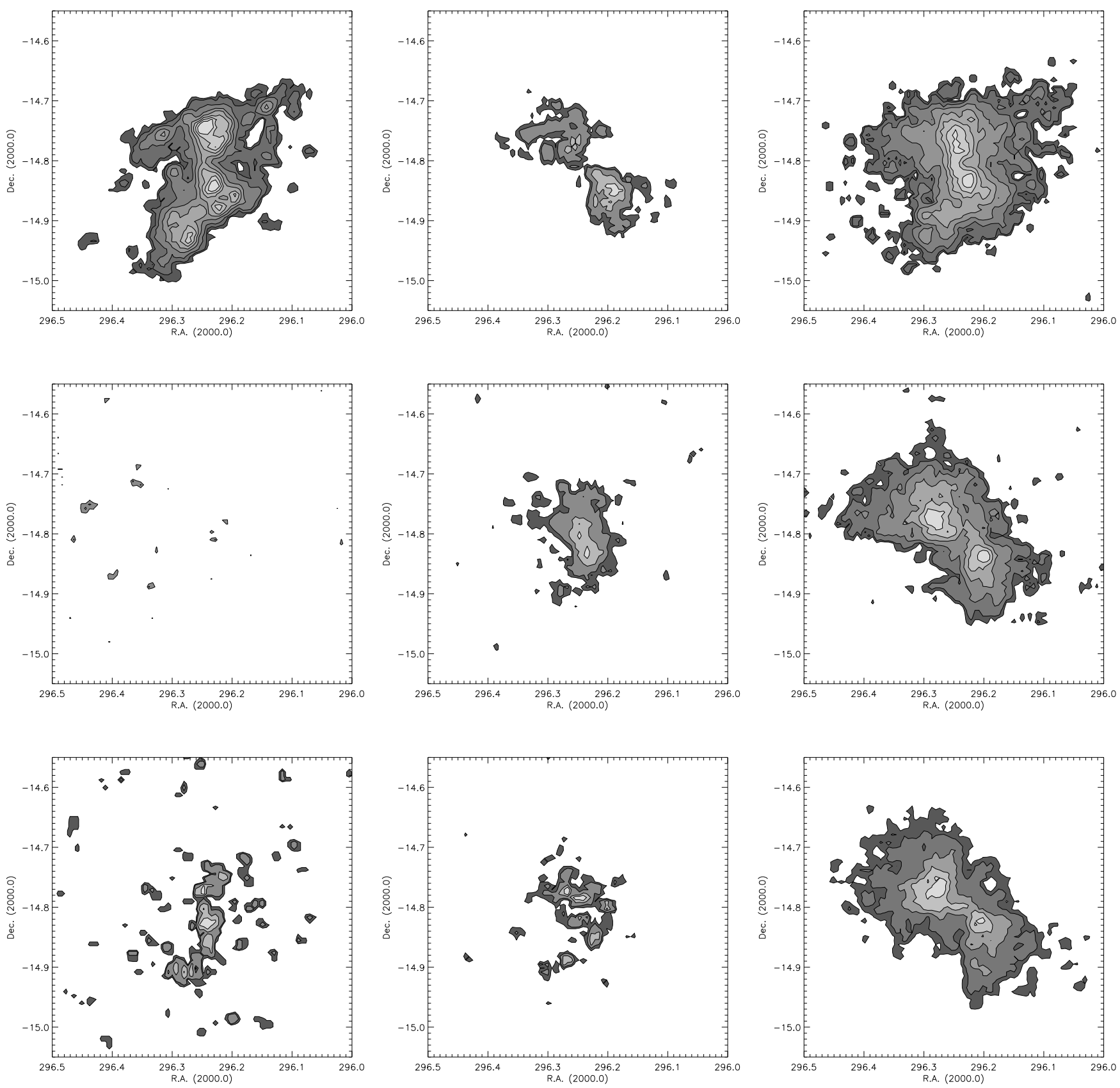

Fig. 7. Spatial distribution of different stellar populations of NGC 6822. Upper: from left to right, CMD regions I, II and III. Middle: from left to right, CMD regions IV, V and VI. Lower: from left to right, CMD regions VII, VIII and IX.

For example, the controversial LMC bimodal structure (Fig. 4) with its peaks centered on $550 \mathrm{pc}$ and $750 \mathrm{pc}$ is probably real.

A few very large structures with size $>900 \mathrm{pc}$ were also found in the MCs, three of them in the LMC and five in the SMC. Such structures were not found in NGC 6822. Whether this is related to some particular property of the galaxies is not clear from this investigation.

\subsection{A possible strong interaction}

The examination of how the different stellar populations are distributed in space reveals two different structures: the N-S barlike structure of young stars and the NE-SW elliptical structure of the old stars. The non-similar spatial distribution of young and old stars has been pointed out by previous studies (Hodge 1977; de Blok \& Walter 2003; Komiyama et al. 2003; Battinelli et al. 2003; Demers et al. 2006). What is not commented on is the poor likelihood of a smooth transition from the younger structure to the older one by stellar dissemination. In a quiescent and undisturbed galaxy, one would expect that the stars, after their creation, would drift and/or participate in the expansion of the OB associations in which they were born. This would probably result in an old population including the observed star forming regions. In the case of NGC 6822, star formation is taking place on a large scale, along the north-south direction. The extended old stellar component, however, does not include the star forming regions, but it has a rather different alignment. This probably means that the huge young structure is too young to have already 
Table 4. Colour, magnitude and age limits and dominant stellar component of the selected colour-magnitude diagram regions.

\begin{tabular}{ccccc}
\hline \hline $\begin{array}{c}\text { Selected } \\
\text { Region }\end{array}$ & $\begin{array}{c}V \\
(\mathrm{mag})\end{array}$ & $\begin{array}{c}(B-V)_{0} \\
(\mathrm{mag})\end{array}$ & $\begin{array}{c}\text { age } \\
(\mathrm{Myr})\end{array}$ & $\begin{array}{c}\text { Stellar } \\
\text { Content }\end{array}$ \\
\hline I & $18.0-23.0$ & $-0.4-0.0$ & $\leq 320$ & $\mathrm{MS}^{a}$ \\
II & $23.0-24.0$ & $-0.4-0.3$ & $\leq 630$ & MS $^{a}$ \\
III & $18.0-23.0$ & $0.0-0.3$ & $60-400$ & Post MS $^{b}$ \\
IV & $15.5-19.5$ & $0.4-0.8$ & $10-80$ & MW stars $^{c}$ \\
V & $19.5-22.0$ & $0.4-0.8$ & $80-320$ & Post MS $^{b}$ \\
VI & $22.0-24.0$ & $0.4-0.8$ & $320-500$ & Post MS $^{b}$ \\
VII & $19.0-20.5$ & $1.3-1.6$ & $130-630$ & MW stars $^{c}$ \\
VIII & $20.5-21.5$ & $1.3-1.6$ & $630-3160$ & MW stars $^{c}$ \\
IX & $21.5-24.0$ & $1.0-2.0$ & $\geq 790$ & RGs $^{d}$ \\
\hline
\end{tabular}

a Main Sequence stars.

${ }^{b}$ Post Main Sequence stars.

${ }^{c}$ Mostly Milky Way stars. The age range in Col. 4 is referring to NGC 6822 stars.

$d$ Red Giants.

produced an old "halo" around it. This very recent and spatially extended star formation, together with the presence of the highly perturbed gas disk, bring up the possibility of a past strong event (Demers et al. 2006). A merging event is a reasonable scenario, given the lack of a nearby massive galaxy to NGC 6822 which could tidally disrupt it after one or more close encounters. Such an event could be responsible for the excess of the recent star formation and the asymmetric gas disk.

Whatever its nature, a strong interaction could have many consequences for our current understanding of NGC 6822. The interaction scenario between NGC 6822 and the NW Cloud (de Blok \& Walter 2000, 2006) could be insufficient to explain such large differences between the young and the old stellar populations. The mass of the Cloud is $\sim 1.4 \times 10^{7} M_{\odot}$ (de Blok \& Walter 2000), only $5 \%$ of the total observed baryonic mass of NGC $6822\left(\sim 2.8 \times 10^{8} M_{\odot}\right.$, Weldrake et al. 2003). The suggestion that the Cloud is a separate galaxy has to be treated with caution. One of the main arguments supporting this is that the NW half of atomic hydrogen contains $20 \%$ more mass than the SE half, as measured with respect to a minor axis passing through the geometrical center. This was based on the assumption of an intrinsically symmetric gas disk (de Blok \& Walter 2000). However, from the same work it is revealed for the first time that the gas disk of NGC 6822 is highly perturbed. Additionally, if NGC 6822 has undergone a strong interaction in the past, the assumption of a symmetric gas disc could be questionable. Whether this gas Cloud is a separate galaxy needs further investigation. The indication of a strong past interaction also strengthens the argument of NGC 6822 being a Polar Ring galaxy (Demers et al. 2006), as it provides the most important prerequisite for its formation.

The young large scale structure of NGC 6822 (Fig. 7) is associated with stars younger than $350 \pm 50 \mathrm{Myr}$, while the old large scale structure (which is misaligned with respect to the young one) is associated with stars older than $350 \pm 50 \mathrm{Myr}$, dating the possible strong interaction before that time. This timescale is in satisfactory agreement with the timescales of the increase of the recent SFR and the formation of the tidal gas features (see Introduction). Furthermore, Demers et al. (2006) place the strong interaction necessary for the formation of the Polar Ring well before $500 \mathrm{Myr}$. The validity of the $350 \pm 50 \mathrm{Myr}$ timescale is also confirmed by the fact that the stars of NGC 6822 probably need 400 Myr or even 500-600 Myr to mix (Wyder 2001).
Thus, the time limit between the young and the old large scale structures is probably unbiased by stellar mixing.

Although from the present study there are indications that NGC 6822 could have undergone a strong and not a weak interaction in the past, it is not possible to derive certain conclusions from our data. Further investigation is needed to obtain a more definite answer.

\section{Conclusions}

We used the "Local Group Survey" stellar catalogue of NGC 6822 to identify its star complexes. These regions were detected from the isopleths based on counts of the young stars above a statistical cutoff limit, resulting in a list of the positions and sizes of star complexes.

Indications of hierarchical star formation were found in NGC 6822, in terms of spatial distribution and time evolution. Star complexes are mainly found inside larger star complexes, and younger star forming structures are clearly less extended than the older ones. These findings add support to the Efremov \& Elmegreen model (Elmegreen \& Efremov 1996; Efremov \& Elmegreen 1998), where star complexes are part of a continuous star formation hierarchy, following the hierarchical distribution of the gas, and star formation proceeds more slowly in larger star forming regions than in smaller ones. Additionaly, indications of hierarchical star formation in terms of preferable sizes of the star complexes were found in NGC 6822 and the Magellanic Clouds. The diagrams of the sizes of all the detected star complexes of NGC 6822 and the Magellanic Clouds, sorted in ascending order, were used along with the two-tailed KolmogorovSmirnov statistical test to search for different size groups: two main groups were identified, the first one ranging from $\sim 150 \mathrm{pc}$ to $\sim 300-400 \mathrm{pc}$ and the second up to $\sim 800 \mathrm{pc}$. This is probably consistent with the empirically extracted hierarchy of stellar aggregates, stellar complexes and stellar supercomplexes. The smallest size groups, like OB associations, remain to be tested as to whether they form a distinct group.

The large scale structures of the NGC 6822 galaxy were studied by selecting stellar populations of various ages from the colour-magnitude diagram and projecting their stellar content in space. It was found that the old population does not contain the young one, but rather they form two distinct structures rotated in position angle. A possible strong interaction could explain this misalignment and, given that NGC 6822 is relatively isolated in space, a merging event could be considered as a reasonable scenario. The traces of the possible strong interaction on the spatial distribution of the stellar populations date to before $350 \pm 50 \mathrm{Myr}$, in agreement with the recent star formation excess of this galaxy.

Acknowledgements. We are grateful to the anonymous referee for comments and suggestions that significally improved this paper. This work was supported by the Special Account for Research Grants of the National and Kapodistrian University of Athens. We would like to thank the Local Group Survey Team for publicly releasing their data. We are also grateful to C. Gallart (Instituto de Astrofísica de Canarias, La Laguna, Spain) who kindly offered her data for tests.

\section{References}

Battinelli, P., Demers, S., \& Letarte, B. 2003, A\&A, 405, 563 de Blok, W. J. G., \& Walter, F. 2000, ApJ, 537, L95 de Blok, W. J. G., \& Walter, F. 2003, MNRAS, 341, L39 
de Blok, W. J. G., \& Walter, F. 2006, AJ, 131, 343

Demers, S., Battinelli, P., \& Kunkel, W. E. 2006, ApJ, 636L, 85

Elmegreen, B. G., \& Efremov, Yu. N., 1996, ApJ, 466, 802

Efremov, Yu. N. 1978, PAZh, 4, 125

Efremov, Yu. N. 1979, PAZh, 5, 21

Efremov, Yu. N. 1987, Ap\&SS, 135, 119

Efremov, Yu. N., \& Elmegreen, B. G., 1998, MNRAS, 299, 588

Gallart, C., Aparicio, A., \& Vilchez, J. M. 1996a, AJ, 112, 1928

Gallart, C., Bertelli, G., \& Chiosi. C. 1996b, AJ, 112, 2596

Girardi, L., Bertelli, G., Bressan, A., et al. 2002, A\&A, 391, 195

Hodge, P. W. 1977, ApJS, 33, 69

Hodge, P. W. 1980, ApJ, 241, 125

Hodge, P. W., Smith, T., Eskridge, P., Mac Gillivray, H., \& Beard, S. 1991, ApJ, 379,621
Komiyama, Y., Sadanori, O., Masafumi, Y., et al. 2003, ApJ, 590, L17

Livanou, E., 2007, Ph.D. Thesis, National and Kapodistrian University of Athens

Livanou, E., Kontizas, M., Gonidakis, I., et al. 2006, A\&A, 451, 431

Livanou, E., Gonidakis, I., Kontizas, et al. 2007, AJ, 133, 2179

Maragoudaki, F., Kontizas, M., Kontizas, E., Dapergolas, A., \& Morgan, D. H. 1998, A\&A, 338, L29

Maragoudaki, F., Kontizas, M., Morgan D. H., et al. 2001, A\&A, 379, 864

Massey, P., Olsen, K. A. G., Hodge, P. W., et al. 2006, AJ, 131, 2478

Massey, P., Olsen, K. A. G., Hodge, P. W., et al. 2007, AJ, 133, 2393

Mateo, M. L. 1998, ARA\&A, 36, 435

Skillman, E. D., Terlevich, R., \& Melnick, J. 1989, MNRAS, 240, 563

Valenzuela, O., Rhee, G., Klypin, A., et al. 2007, ApJ, 657, 773

Weldrake, D. T. F., de Blok, W. J. G., \& Walter, F. 2003, MNRAS, 340, 12

Wyder, T. K. 2001, AJ, 122, 2490 